\title{
Measurements of $\tau$ hadronic branching fractions and spectra, and search for second class current $\tau$ decays at BaBar
}

\section{Roger Barlow*Huddersfield University}

E-mail: R.Barlow@hud.ac.uk

\begin{abstract}
We present a set of preliminary and final results on tau branching fractions and invariant mass spectra, and a search for the second class current decay $\tau \rightarrow \pi \eta^{\prime}(958) v_{\tau}$. The results include new studies of a large number 3 and 5-prong tau decay modes either inclusive or classified according the hadronic resonance content $\left(K_{S}^{0}, \eta, \omega\right.$, etc.). All results are based on the analysis of the complete $B A B A R$ data-set consisting on 430 million $\tau$ lepton pairs, corresponding to an integrated luminosity of $468 \mathrm{fb}^{-} 1$, collected with the BABAR detector at the PEP-II asymmetric energy $e^{+} e^{-}$ storage rings.
\end{abstract}

The European Physical Society Conference on High Energy Physics 18-24 July, 2013

Stockholm, Sweden

\footnotetext{
* Speaker.
} 


\section{Introduction}

Although the PEP-II storage ring was designed as a factory for B mesons, its high luminosity made it an excellent machine for the production of $\tau$ leptons. The results presented here are based on up to 430 million $\tau$ pairs - almost one billion $\tau$ leptons - in the complete BABAR dataset. Such $\tau$ studies serve as a testbed for models of mesons, and for QCD in general.

The BABAR detector has been described elsewhere[1]. A particular feature of these $\tau$ analyses is the powerful $\pi / K$ discrimination provided by the DIRC[2] and also by $d E / d x$ measurements in the tracking chambers. The analyses presented here differ from each other in detail, but share use a similar broad technique: the event is divided in two using the thrust axis in the centre of mass. One hemisphere is used to tag the event as a $\tau$, by requiring that it contain a single charged track. Further insisting that this track be identified as a lepton (electron or muon) increases the purity of the sample at the price of a lower efficiency.

\section{2. $\tau$ to 3 charged particles}

We have selected 3-prong $\tau$ decays - a 'prong' means any charged particle track, in this analysis they are either pions or kaons - and obtained branching ratios for the decays $\tau^{-} \rightarrow \pi^{-} \pi^{+} \pi^{-} v_{\tau}$, $K^{-} \pi^{+} \pi^{-} v_{\tau}, K^{-} K^{+} \pi^{-} v_{\tau}$, and $K^{-} K^{+} K^{-} v_{\tau}$. These have already been published[3]. Here also present (as preliminary results) the mass distributions for these channels.
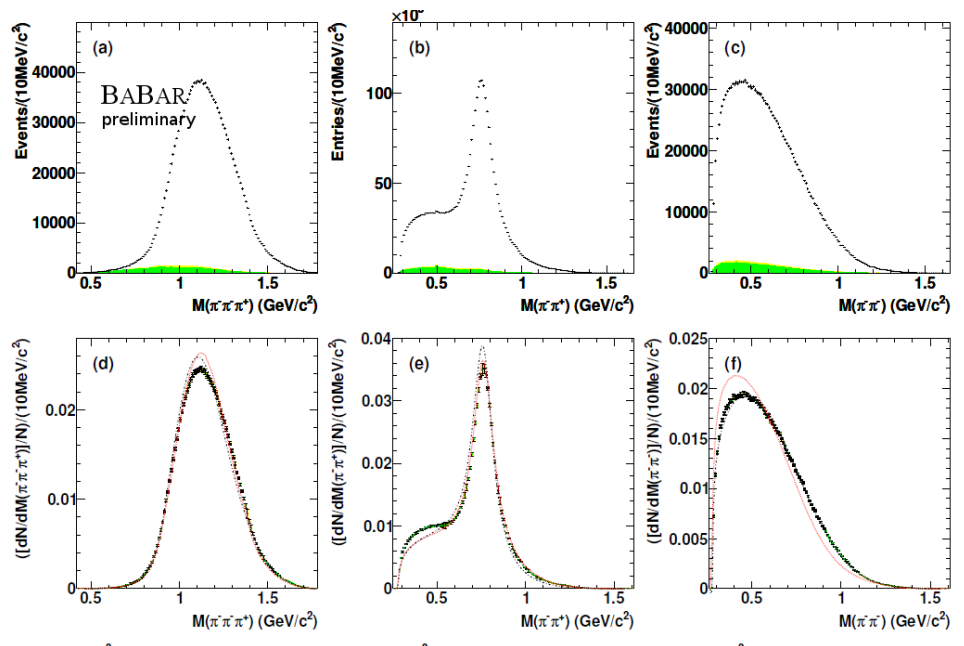

Figure 1: Mass combinations in $\pi^{-} \pi^{+} \pi^{-}$decays

Figure 1 presents the mass combinations of the three pions. The top row (a-c) gives the measured distributions for the $3 \pi$ mass, the unlike-sign and like-sign $2 \pi$ combinations. The small estimated backgrounds, which are from other $\tau$ decays, are shown in green.

The bottom row (d-f) presents the unfolded distributions, with the backgrounds subtracted and the resultion unfolded using a Bayesian technique[4]. The curves show the predictions of the Tauola Monte Carlo [5]. This is the current default tune, version 2.8, based on measurments at CLEO. The agreement is generally very good, though not perfect. This decay is basically modelled as occurring through the $a_{1}(1260)$, which in turn decays through the channel $a_{1} \rightarrow \rho \pi$. 

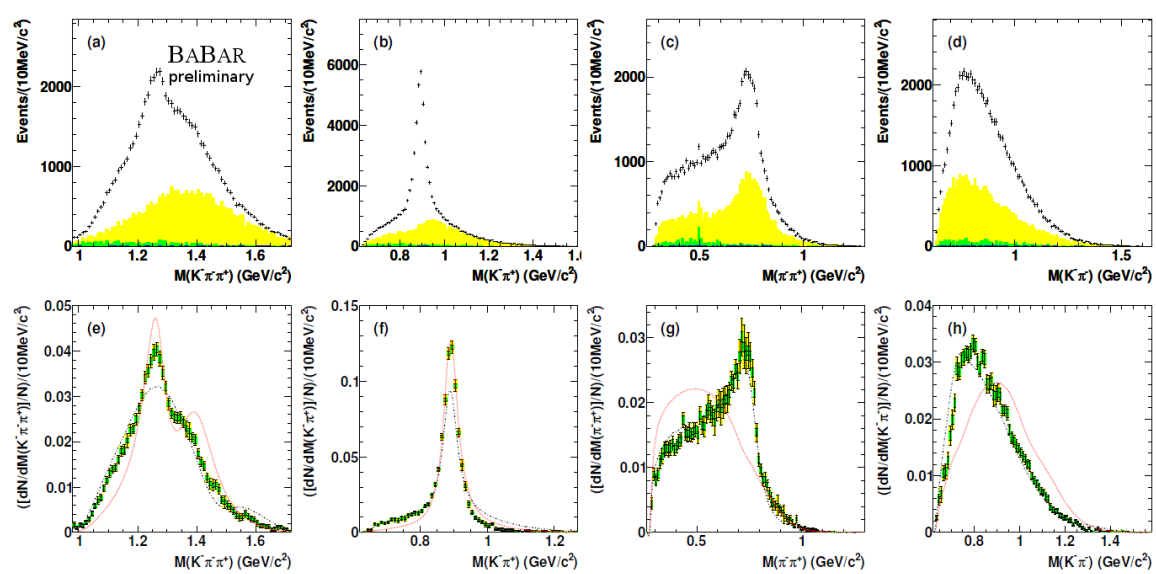

Figure 2: Mass combinations in $K^{-} \pi^{+} \pi^{-}$decays

Figure 2 shows the mass combinations for the $K \pi^{+} \pi^{-}$decays. Again, the top row shows the measured distributions and the lower the unfolded distributions. The background from other $\tau$ decays (green) is again small, but there are now also contributions from cross-feed from the $3 \pi$ decay, shown in yellow.

Our results agree with those of Belle[6] except in the $K \pi \pi$ combination in the region around $1.4 \mathrm{GeV}$, where the shape is dominated by the background subtraction.

The agreement with the Tauola 2.8 predictions (the red curves) is not good. We have re-tuned the parameters and obtained better agreement, as shown in the black curves.
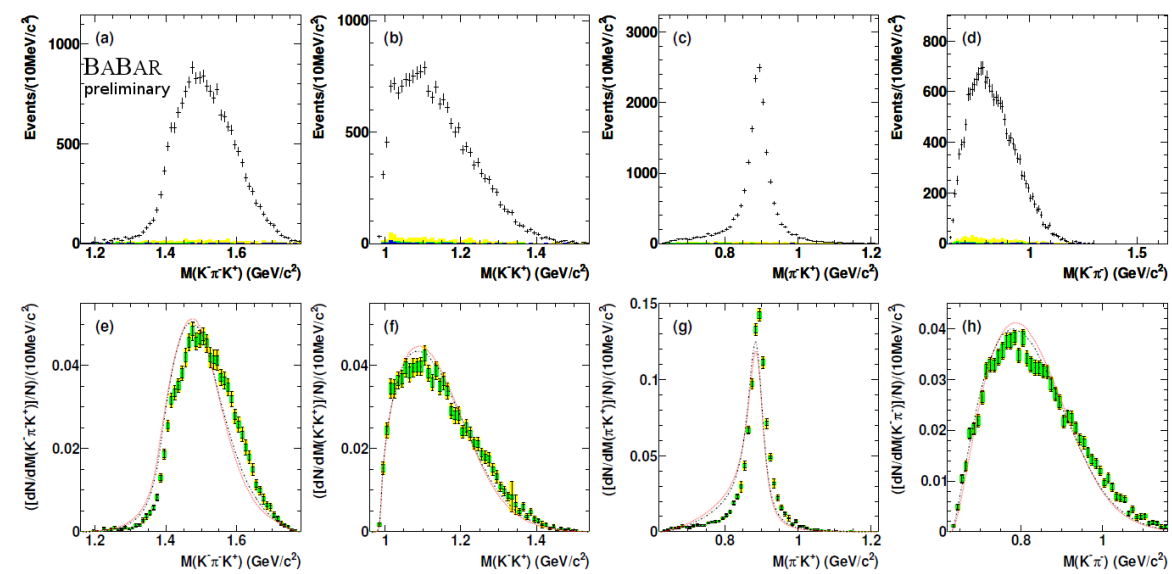

Figure 3: Mass combinations in $K^{-} K^{+} \pi^{-}$decays

Figure 3 shows the mass combinations for the $K^{+} K^{-} \pi^{-}$combinations. The inner error bars (green) show the statistical uncertainty, and the outer (yellow) ones have the systematic uncertainty added in quadrature. We have again retuned the Monte Carlo parameters, though the standard Tauola predictions are good. The decay is dominated by the channel $K^{-} K^{* 0}(892)$ 

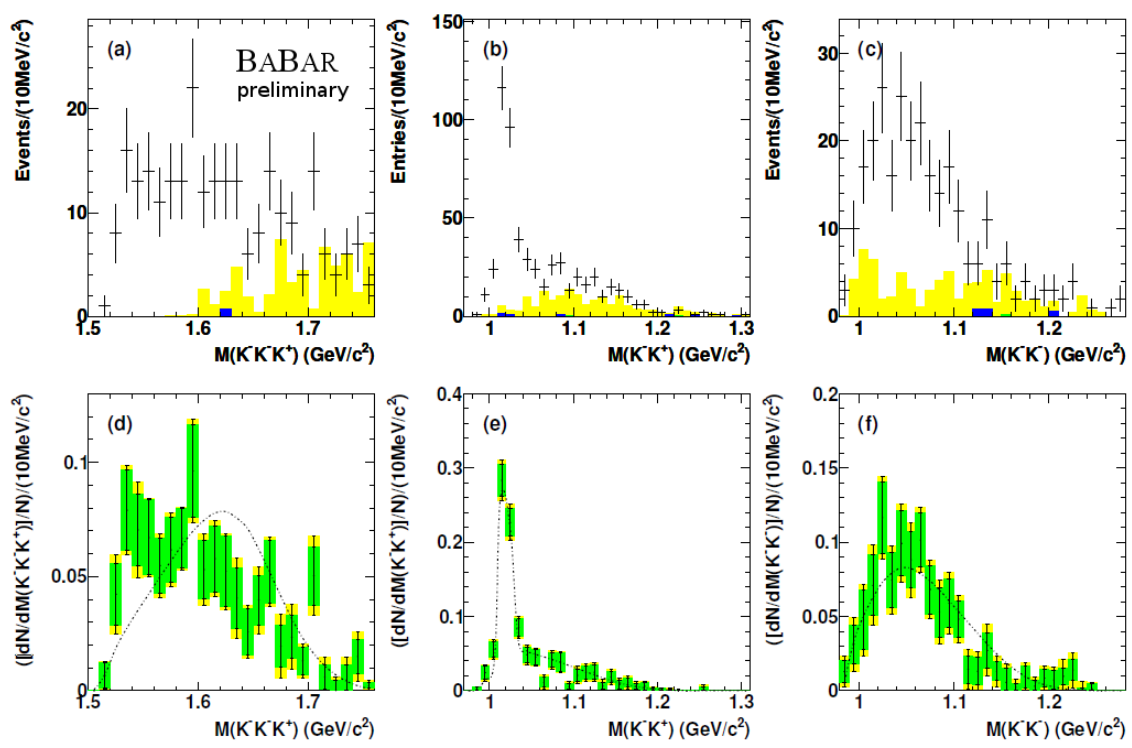

Figure 4: Mass combinations in $K^{-} K^{+} K^{-}$decays

Figure 4 shows the mass distributions for 3 kaons. Here the dominant channel is $K^{-} \phi$

\section{3. $\tau$ to 5 prongs}

We have analysed a set of high multiplicity decays of the $\tau$ through a number of channels involving pions and multi-pion resonances[7]. The three intermediate states $\tau^{-} \rightarrow \pi^{-} f_{1} v_{\tau}, \tau^{-} \rightarrow$ $(3 \pi)^{-} \eta v_{\tau}$, and $\tau^{-} \rightarrow(3 \pi)^{-} \omega v_{\tau}$ produce the four final states $\pi^{-} \pi^{-} \pi^{-} \pi^{+} \pi^{+}, \pi^{-} \pi^{+} \pi^{-} \eta(\gamma \gamma)$, $\pi^{-} \pi^{+} \pi^{-} \pi^{0} \pi^{0} \pi^{0}$, and $\pi^{-} \pi^{-} \pi^{-} \pi^{+} \pi^{+} \pi^{0}$ by various routes:

The $f_{1}$ may decay to $\pi^{+} \pi^{-} \eta$ or $\pi^{+} \pi^{-} \pi^{+} \pi^{-}$.

The $\eta$ may decay to $\pi^{+} \pi^{-} \pi^{0}$ or $3 \pi^{0}$ or $\gamma \gamma$

The $\left(3 \pi^{-}\right)$system may be $\pi^{-} \pi^{+} \pi^{-}$or $\pi^{-} \pi^{0} \pi^{0}$

The two different decay modes of the $f_{1}$ are clearly seen, including the three different decay modes of the $\eta$. These are shown in figure 5. A fit to the data give the world's best value for the mass of the $f_{1}$ as $1.28116 \pm 0.00039 \pm 0.00045 \mathrm{GeV} / \mathrm{c}^{2}$.
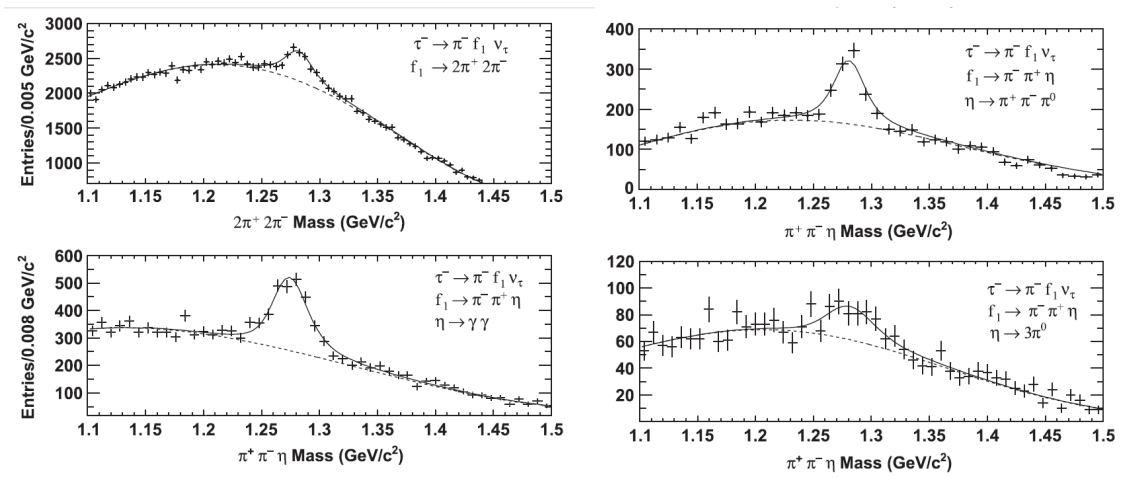

Figure 5: Decays of the $f_{1}$ meson 
Results for these branching ratios are shown in table 1 . The branching ratios for the two $f_{1}$ decays are presented separately. If we take the ratio then we obtain (using isospin symmetry)

$$
\frac{\mathscr{B}\left(f_{1} \rightarrow \pi^{+} \pi^{-} \pi^{+} \pi^{-}\right)}{\mathscr{B}\left(f_{1} \rightarrow \pi \pi \eta\right)}=0.28 \pm 0.02 \pm 0.02
$$

which agrees with the average value of $0.41 \pm 0.14$ obtained by the Particle Data Group, but not with their fitted value of $0.63 \pm 0.06[8]$.

\begin{tabular}{|l|l|}
\hline Channel & Branching Ratio \\
\hline$\pi^{-} \pi^{+} \pi^{-} \eta v_{\tau}$ & $(2.25 \pm 0.07 \pm 0.12) \times 10^{-4}$ \\
$\pi^{-} \pi^{0} \pi^{0} \eta v_{\tau}$ & $(2.01 \pm 0.34 \pm 0.22) \times 10^{-4}$ \\
$\pi f_{1}, v_{\tau}, f_{1} \rightarrow \pi^{+} \pi^{-} \pi^{+} \pi^{-}$ & $(5.20 \pm 0.31 \pm 0.37) \times 10^{-5}$ \\
$\pi f_{1}, v_{\tau}, f_{1} \rightarrow \pi^{+} \pi^{-} \eta$ & $(1.26 \pm 0.06 \pm 0.06) \times 10^{-4}$ \\
$\pi^{-} \pi^{+} \pi^{-} \omega v_{\tau}$ & $(7.3 \pm 1.2 \pm 1.2) \times 10^{-5}$ \\
$\pi^{-} \pi^{0} \pi^{0} \omega v_{\tau}$ & $(7.8 \pm 0.04 \pm 0.40) \times 10^{-4}$ \\
$\pi^{-} \pi^{+} \pi^{-} \pi^{+} \pi^{-} v_{\tau}$ non-resonant & $(3.6 \pm 0.3 \pm 0.9) \times 10^{-5}$ \\
$\pi^{-} \pi^{+} \pi^{-} \pi^{+} \pi^{-} \pi^{0} v_{\tau}$ non-resonant & $<5.8 \times 10^{-5} @ 90 \% \mathrm{CL}$ \\
$\pi^{-} \pi^{+} \pi^{-} \pi^{0} \pi^{0} \pi^{0} v_{\tau}$ non-resonant & $<4.0 \times 10^{-6} @ 90 \% \mathrm{CL}$ \\
\hline$\pi^{-} \eta^{\prime} v_{\tau}$ & $<1.2 \times 10^{-5} @ 90 \% \mathrm{CL}$ \\
$\pi^{-} \eta^{\prime} v_{\tau} \pi^{0}$ & $<2.4 \times 10^{-6} @ 90 \% \mathrm{CL}$ \\
$K^{-} \eta^{\prime} v_{\tau}$ & $<2.4 \times 10^{-6} @ 90 \% C L$ \\
\hline$K^{-} \pi^{+} \pi^{-} \pi^{+} \pi^{-} v_{\tau}$ & $<5.0 \times 10^{-6} @ 90 \% C L$ \\
$K^{+} \pi^{-} \pi^{-} \pi^{+} \pi^{-} v_{\tau}$ & $<4.5 \times 10^{-7} @ 90 \% C L$ \\
$K^{-} K^{+} \pi^{-} \pi^{+} \pi^{-} v_{\tau}$ & $<1.9 \times 10^{-6} @ 90 \% C L$ \\
$K^{-} \pi^{+} \pi^{-} \pi^{+} \pi^{-} \pi^{0} v_{\tau}$ & $<8 \times 10^{-7} @ 90 \% C L$ \\
$K^{+} \pi^{+} \pi^{-} \pi^{+} \pi^{-} \pi^{0} v_{\tau}$ & \\
\hline
\end{tabular}

Table 1: Values and limits of branching ratios for high multiplicity tau decay channels

We also searched for decays involving the $\eta^{\prime}$ meson, in its decays to $\pi^{+} \pi^{-} \eta$, again using all three $\eta$ decay channels. A peak at the $\eta^{\prime}$ mass of $958 \mathrm{MeV}$ is seen in the data, but it is compatible with the expected background from $q \bar{q}$ events. The decay $\tau^{-} \rightarrow \pi^{-} \eta^{\prime} v_{\tau}$ is a Second Class current[9]. Such currents are forbidden by isopsin/G-parity considerations or, equivalently, in the limit that the $u$ and $d$ quarks have equal mass. As this equality is not exact, the Standard Model predictions for such currents are small but non-zero, and we are now probing the values at which a signal might be expected,so this channel, and also the decay $\tau^{-} \rightarrow \pi^{-} \omega \nu_{\tau}$ channel [10] are of great interest. We have the world's best limits, but still have not observed a signal.

\section{4. $\tau^{-}$decays with a 1 prong plus two $\mathrm{V}$ topology.}

$\mathrm{K}_{s}^{0}$ particles from $\tau$ decays typically travel a short distance in the BABAR tracking chambers before decaying to two pions, giving (for the $\pi^{+} \pi^{-}$decay mode) two charged tracks which intersect at a point away from the beam spot, with the invariant mass of the $\mathrm{K}_{s}^{0}$.

We have analysed $\tau$ decays with two such $\mathrm{K}_{s}^{0}$ decays, plus a possible additiional $\pi^{0}$ [11]. A $\mathrm{K}_{s}^{0}$ is selected as a pair of oppositely charged tracks which does not point to the beam spot, whose 
vertex is at least 3 sigma displaced from it, and whose invariant mass lies between 0.475 and 0.525 $\mathrm{Gev} / \mathrm{c}^{2}$.

The numbers of events seen where the single prong is identified as a kaon rather than a pion are consistent with expected backgrounds (which come dominantly from the $1 \%$ probablity of a $\pi$ being identified as a K), and we use them to give $90 \%$ confidence level upper limits.

Again, the standard Tauola package[5] does a good but not perfect job of describing the observed mass distributions. This is accounted for by re-weighting simulated events (needed for the calculation of efficiencies) according to the observed distribution in the two dimensional plot of the $\pi^{-} \mathrm{K}_{s}^{0}$ masses. This is presented in Figure 6, which shows how the $\pi^{-} \mathrm{K}_{s}^{0} \mathrm{~K}_{s}^{0}$ state can be well described as going through the $K^{0} K^{*}(892)$ channel

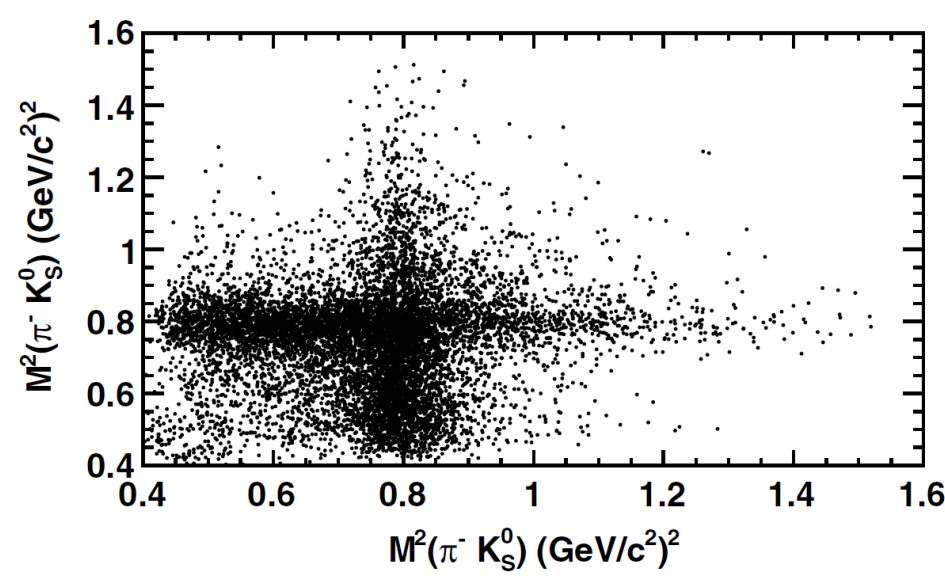

Figure 6: Mass plot of the two $\pi^{-} K_{s}^{0}$ combinations

Results are shown in table 2.

\begin{tabular}{|l|l|}
\hline Channel & Branching Ratio \\
\hline$\tau^{-} \rightarrow \pi^{-} \mathrm{K}_{s}^{0} \mathrm{~K}_{s}^{0} v_{\tau}$ & $(2.13 \pm 0.04 \pm 0.08) \times 10^{-4}$ \\
$\tau^{-} \rightarrow \pi^{-} \mathrm{K}_{s}^{0} \mathrm{~K}_{s}^{0} \pi^{0} v_{\tau}$ & $(1.60 \pm 0.20 \pm 0.22) \times 10^{-5}$ \\
$\tau^{-} \rightarrow \mathrm{K}^{-} \mathrm{K}_{s}^{0} \mathrm{~K}_{s}^{0} v_{\tau}$ & $<6.3 \times 10^{-7} @ 90 \% \mathrm{CL}$ \\
$\tau^{-} \rightarrow \mathrm{K}^{-} \mathrm{K}_{s}^{0} \mathrm{~K}_{s}^{0} \pi^{0} v_{\tau}$ & $<4.0 \times 10^{-7} @ 90 \% \mathrm{CL}$ \\
\hline
\end{tabular}

Table 2: Results

The precision of these measurements is a significant improvement on previous ones. They are interesting not only in their own right, but also as an important source of background to the $\tau^{-} \rightarrow \pi^{-} \mathrm{K}_{s}^{0} v_{\tau}$ decay channel, which is sensitive to new physics and will provide an important measurement at any future B factory.

\section{Conclusions}

The $B A B A R$ dataset is still providing a rich source of results on decays ot the $\tau$. Further analyses are in progress and will be reported at future conferences. 


\section{References}

[1] B. Aubert et al., (BABAR collaboration) Nucl. Instrum. \& Meth. A479, 1, (2002)

[2] I. Adam et al., Nucl. Instrum. \& Meth. A538, 281, (2005)

[3] B. Aubert et al., (BABAR collaboration) Phys. Rev. Lett. 100, 011801, (2008)

[4] G. D’Agostini, Nucl. Instrum. \& Meth. A362, 487, (1995)

[5] S. Jadach, Z. Wa̧s, R. Decker and J. Kühn, Comput. Phys. Commun. 76 361, (1993)

[6] M. Lee et al.,(Belle collaboration) Phys.Rev. . D81, 113007, (2010)

[7] J. P. Lees et al., (BABAR collaboration) Phys. Rev. D 86,092010, (2012)

[8] J. Beringer et al.,(Particle Data Group), Phys. Rev. D 86 010001, (2012)

[9] S. Weinberg, Phys. Rev. 112, 1375, (1958)

[10] B. Aubert et al.,(BABAR collaboration) Phys. Rev. Lett. 103041802, ,2009)

[11] J. P. Lees et al.,(BABAR collaboration) Phys. Rev. D 86,092013, (2012) 